\title{
émulations
}

\section{Jean-Louis Fabiani - Pierre Bourdieu. Un structuralisme héroïque}

\section{Bastien Fond}

Émulations - Revue de sciences sociales

2018, «Comptes rendus critiques, En ligne»

\section{Article disponible à l'adresse suivante}

https://ojs.uclouvain.be/index.php/emulations/article/view/7343

\section{Pour citer cet article}

Bastien Fond, «Jean-Louis Fabiani — Pierre Bourdieu. Un structuralisme héroïque », Émulations, en ligne. Mise en ligne le 4 janvier 2018.

DOI : 10.14428/emulations.cr.035

Distribution électronique : Université catholique de Louvain (Belgique) : ojs.uclouvain.be

(C) Cet article est mis à disposition selon les termes de la Licence Creative Commons Attribution, Pas d'Utilisation Commerciale 4.0 International. http://creativecommons.org/licenses/by-nc/4.0/

Éditeur : Émulations - Revue de sciences sociales / Presses universitaires de Louvain https://ojs.uclouvain.be/index.php/emulations

ISSN électronique : 1784-5734

UCL PRESSES

UNIVERSITAIRES 


\title{
Jean-Louis Fabiani - Pierre Bourdieu. Un structuralisme héroïque
}

\begin{abstract}
Bastien Fond ${ }^{1}$
Recensé : Jean-Louis Fabiani, Pierre Bourdieu. Un structuralisme héroïque, Paris, Seuil ( «a couleur des idées »), 2016, 312 p.

Un peu plus d'une décennie après la mort de Pierre Bourdieu, Jean-Louis Fabiani revient sur l'œuvre monumentale de son ancien collègue. Prenant l'héroïsme pour motclé, la vocation de l'ouvrage Pierre Bourdieu. Un structuralisme héroïque n'est pourtant pas de délivrer un témoignage glorificateur. Il s'agit plutôt de décrypter le mythe de la figure bourdieusienne au fil d'un commentaire érudit de litinéraire intellectuel du penseur. S'il fallait assigner un parti-pris à Fabiani, c'est celui d'une explication génétique : reconstituer le processus de maturation des concepts pour les expliciter par leur genèse et éclaircir ainsi leur réception. Avant tout, cet essai est une reconquête didactique de l'ésotérisme qui gît derrière l'exotérisme bourdieusien² ${ }^{2}$.

Comme le rappelle l'auteur à propos de la notion bourdieusienne de capital : souvent «l'effort de clarification opéré par Bourdieu a ajouté à la complexité du concept, qui se laisse de plus en plus difficilement décomposer » (p. 111). Pour paraphraser Fabiani, plus on s'enfonce dans le corpus bourdieusien, plus la « magie sociale » (p. 110) de cette pensée opère, jusqu'à transformer la théorie en «boîte noire » (p. 83). Comme dans Les Confessions de Rousseau (2011), où un cœur «transparent comme le cristal» (p. 527) esquisse des intentions qui ne peuvent être avouées qu'à demi-mot parce que la matérialité du minerai reste un obstacle à sa transparence (Lejeune, 1973), dans le cas de Bourdieu, le succès de ses thèses a montré que ses textes traduisent autant qu'ils trahissent ses intuitions. Fabiani parle d'« usages nettement péjoratifs que la lecture rapide de Bourdieu a diffusés dans le langage ordinaire » (p. 228). Ses textes communiquent une vision du monde social si forte qu'elle est sujette à toutes formes de récupérations — allusives ou abusives? — par son lectorat : «c'est un lexique, plus qu'une méthode, que ses héritiers valorisent sur le marché » (p. 100).
\end{abstract}

${ }^{1}$ Diplômé de l'Institut d'études politiques de Lille (mention philosophie, politique et économie).

${ }^{2}$ Bourdieu y est associé à « une tradition en France [...] qui articule de manière complexe l'ésotérisme, qui naît de la nécessité de garantir le fonctionnement autonome d'une science, et l'exotérisme, qui exige que le scientifique puisse se faire comprendre de tous » (p. 132). 
Dans ces conditions, Fabiani construit son développement de manière à « réintégrer le sociologue dans le cadre analytique qu'il a lui-même construit »(p. 14). Les trois premiers chapitres explorent le corpus bourdieusien de manière synoptique et sont consacrés respectivement à chacun des concepts clés de Bourdieu : le champ, l'habitus, puis le capital (sans négliger pour autant la complexité d'un appareillage conceptuel qui repose aussi sur l'illusio et l'hystérésis). Quant aux chapitres 4, 5 et 6, ils sont dédiés à une approche macroscopique qui entreprend de recontextualiser la démarche bourdieusienne pour mieux dégager ses apports et sa singularité. In fine, Fabiani boucle son triptyque en confrontant l'originalité de la pensée à celle du penseur pour redonner corps à son récit dans les trois derniers chapitres de l'ouvrage.

Tout au long de sa carrière universitaire, Bourdieu a publié des études de cas qui caractérisent des champs et s'additionnent pour former une théorie éponyme de ces champs. Sous sa plume, « le cas est à la fois particulier et paradigmatique» (p. 35). Dans un effort dialectique, l'universalité de sa théorie des champs repose sur l'exemplarité des cas pratiques examinés. À cet égard, Fabiani rappelle que chaque cas y dévoile des positions déterminées par la structure de l'espace occupé; et même si les différents espaces positionnels décrits par le sociologue sont incomparables, ils ont en commun de produire des effets de structure sur les agents. En termes bourdieusiens, ces effets de champ sont éprouvés par les agents sous forme d'habitus à acquérir pour jouer le jeu «d'une homologie entre les structures mentales de l'habitus individuel et les structures objectives du monde social» (p. 64).

Après la réinterprétation sociologique de la notion de champ, Fabiani s'attaque alors à la reprise de l'habitus grec pour envisager la dimension préconsciente que Bourdieu prête à cette " force formatrice d'habitudes » (p. 76), mais aussi le couple antithétique qu'elle forme avec la notion d'hystérésis. Cette dernière, «empruntée à la physique pour décrire la persistance d'un phénomène alors même que cesse la cause qui l'a produit » (p. 81) permet aussi de penser le désajustement des pratiques sociales pour rétablir de l'indétermination dans le structuralisme bourdieusien. Il importe à Fabiani de rappeler que Bourdieu ne plaçait pas la sociologie que du côté des stratégies gagnantes. Même si l'accumulation d'un capital (culturel, économique, etc.) permet d'optimiser le rendement des activités associées à ce capital, tout champ demeure un espace de luttes imprévisibles.

L'auteur nous met en garde : dans la théorie des champs, le capital ne confère pas un pouvoir infaillible sur le champ. Mais il n'est pas non plus de l'ordre de la simple compétence - contrairement à ce qu'ont pu faire penser certains réinvestissements faussement bourdieusiens (Molénat, 2015). Le propre du capital bourdieusien est de produire un effet de pouvoir. C'est presque une notion spectrale, qui plane sur les rapports de force. D'ailleurs, la notion de capital symbolique - emblématique de la retraduction bourdieusienne du capital en termes d'espèce convertible - est en elle-même fallacieuse. À cet égard, Fabiani ne manque pas de rappeler qu'il vaut mieux y privilégier la 
formulation d'« effets symboliques du capital » qui figure dans les écrits tardifs de Bourdieu (1997).

Finalement, l'objet de cette première partie est donc de dégager l'acception bourdieusienne de concepts qui, certes, précédent le penseur et lui succèdent, mais revêtent surtout une signification tout à fait particulière dès lors qu'ils s'appliquent au monde social tel que Bourdieu le conçoit. Comme le souligne Fabiani, « c'est dans la réitération obsessionnelle d'un vocabulaire qu'une personnalité conceptuelle se construit» (p. 201).

Dans un second temps, l'auteur rappelle qu'au moment où Bourdieu énonce les grands principes de sa théorie, Max Weber est encore très peu traduit en français, et la pensée d'Émile Durkheim est dépréciée. Ces deux éléments expliquent en partie pourquoi, aux yeux de Fabiani, notre homme fait figure de refondateur de la sociologie: «Bourdieu reste un pionnier dans un domaine qui évolue lentement » (p. 138). Sa conception structurale de la causalité en sciences sociales lui permet d'embrasser une pluralité de déterminants sociaux dans ses études de cas à une époque où la sociologie des variables préfère s'attarder sur les effets purs de facteurs indépendants. De plus, sa formation de philosophe l'invite à porter un regard critique sur les grilles d'analyse statistiques pour observer comment les données se construisent avant d'en tirer des conclusions.

À partir des années 1970, il privilégie d’ailleurs les modélisations qui présentent des affinités électives avec la pensée spatiale en termes de champ — sans exclure pour autant les dynamiques temporelles, relève Fabiani. Même si « l'histoire s'efface devant le modèle » (p. 175), elle point toujours à l'horizon, dans des décalages structuraux au service d'une théorie qui tient compte de «la désynchronisation permanente des différents niveaux de la structure » (p. 168). L'événement endosse alors un statut particulier : celui-ci « n'y reste qu'une abstraction logée dans un pli de la structure » (p. 172). C'est au vocabulaire deleuzien que semble ici recourir Fabiani pour articuler son interprétation du système bourdieusien autour d'une malléabilité structurale qui s'informe des évènements (Deleuze, 1988).

La structure argumentative bourdieusienne laisse peu de place à la transgression, il est vrai. Mais Fabiani souligne que c'est parce qu'elle est elle-même transgressive : elle défait les certitudes en offrant « un réseau de contraintes logiques qui peuvent conduire le lecteur à ne pas tomber dans les pièges que tend le langage ordinaire » (p. 202). Ces contraintes logiques, ce sont les termes du jargon bourdieusien (allodoxia, illusio, etc.), qui invitent à penser la réalité autrement pour mieux la dévoiler.

Dans le sillage de cette entreprise de dévoilement, Fabiani ajoute dans un dernier temps que « l'intervention publique et le militantisme sont devenus le cœur de l'activité des sciences sociales » (p. 213) telles que Bourdieu les pratique. Conformément au sens qui s'est forgé pendant la III république, il est un intellectuel — c'est-à-dire « qu'il utilise son "magistère" ou sa position pour communiquer et défendre ses idées et ses convictions » (Yon, 2010 : 204). Non seulement il a institutionnalisé la sociologie en tant 
que discipline autonome en France, mais il en a aussi fait un outil de revendication et n'hésite pas à fustiger les littérateurs (Bernard-Henri Lévy, Philippe Sollers, etc.) au nom de sa ${ }^{3}$ science. D’après Fabiani, « le combat est d'ailleurs le meilleur moyen pour les universitaires de constituer la question de la compétence certifiée comme objet central » (p. 238).

Par ailleurs, cette compétence certifiée par la notoriété dont jouissait Bourdieu à la fin de sa vie lui sert également à dénoncer la misère du monde (Bourdieu, 1993), au moment où ses analyses sociologiques prennent selon Fabiani un tournant émotionnel. Bourdieu n'a pas été qu'à la croisée de la science et de la politique. L’auteur rappelle qu'il a aussi flirté avec la littérature - parfois même au prix de «la disparition de la sociologie » (p. 261).

En définitive, le Bourdieu de cet essai incarne l'oxymore du « héros savant » (p. 278): comme Manet ou Heidegger, c'est en tant que mandarin que le sociologue peut légitimer la «constitution d'un espace transgressif » (p. 290). Dans ce récit, son structuralisme apparaît donc d'autant plus héroïque que la vie du penseur offre la démonstration pratique de ce qu'il théorise autour des héros de ses textes (Bourdieu, 1988 ; 2013). Comme le dit Fabiani, les champs célèbrent aussi des nomothètes ${ }^{4}$ capables de «mâ̂triser toutes les lois du champ pour le subvertir » (p. 284).

L'ouvrage se referme sur cette ambition de délivrer une « sociologie de la vie intellectuelle » (p. 299). Fabiani rappelle d'ailleurs qu'il laisse à d'autres le soin d'élucider la philosophie bourdieusienne. Il renvoie volontiers au travail de Claude Gautier (2012), ou élude la piste des influences freudiennes, sur laquelle Laurent Perreau (2015) s'est engagé. Ce qui l'intéresse, c'est de retrouver l'intellectuel en chair et en os. Et la richesse des chapitres, qui ne commentent pas le projet bourdieusien uniquement sous l'angle d'une étude génétique, nous pousserait presque à qualifier cet essai de biographie intellectuelle. Parfois, le plan tripartite de l'auteur fait primer la narration sur l'exploration des concepts ${ }^{5}$, qui se façonnent à la faveur d'une ligne directrice - le structuralisme héroïque - privilégiant l'exégèse par rapport à une présentation strictement doxographique. Néanmoins, pour reprendre sa propre formule, «l'interaction est susceptible d'être modifiée par le compte rendu [...] que nous en faisons » (p. 294). C'est

\footnotetext{
${ }^{3}$ Le pronom possessif est ici employé pour souligner que, dans l'ouvrage de Fabiani, Bourdieu est surtout présenté comme un défenseur de sa propre manière de faire de la science.

${ }^{4}$ Dans une filiation athénienne, ces législateurs des champs ont acquis suffisamment de pouvoir pour orienter les pratiques dans leur sens.

${ }^{5}$ Comme nous le faisions remarquer dans la présentation du plan de l'ouvrage, Fabiani a préféré une présentation du système bourdieusien plutôt synoptique qu'encyclopédique, et dans laquelle certains concepts sont par conséquent abordés en filigrane.
} 
pourquoi nous invitons à lire directement l'ouvrage de Fabiani pour apprécier ses intuitions — qui sauront en éclairer plus d'un dans leur compréhension de Bourdieu.

\section{Bibliographie}

Bourdieu P. (1988), L’Ontologie politique de Martin Heidegger, Paris, Les Éditions de Minuit ( Le sens commun »).

BouRdieu P. (1993), La Misère du monde, Paris, Seuil (« Libre examen »).

BouRdiEu P. (1997), Méditations pascaliennes, Paris, Seuil («Liber »).

BouRdieu P. (2013), Manet, une révolution symbolique, Paris, Seuil (« Raisons d’agir »).

Deleuze G. (1988), Le Pli. Leibniz et le Baroque, Paris, Les Éditions de Minuit (« Critique »).

GAUTIER C. (2012), La Force du social, Paris, Cerf (« Passages »).

LejeUnE P. (1973), « La punition des enfants. Lecture d’un aveu de Rousseau », Littérature, vol. 10, $n^{\circ}$ 2, p. 31-56.

Molenat X. (2015), «Pierre Bourdieu (1930-2002) : une pensée toujours à l'œuvre», Sciences Humaines, Inédit web. En ligne, consulté le 22 avril 2017. URL: https://www.scienceshumaines.com/pierre-bourdieu-1930-2002-une-penseetoujours-a-l-oeuvre_fr_28372.html.

PeRREAU L. (2015), «Sociologie, psychanalyse et phénoménologie: la théorie de l'inconscient chez Pierre Bourdieu », in M. Gyemant, D. PopA (dir.), Approches phénoménologiques de l’inconscient, Hildesheim, Georg Olms Verlag (« Europaea Memoria »), p. 119-138.

RousSEAU J.-J. (2011), Les Confessions, Paris, Classiques Garnier (« Bibliothèque du XVIII siècle »).

Yon J.-C. (2010), Histoire culturelle de la France au XIX siècle, Paris, Armand Colin (« U : Histoire»). 